\title{
COMPARAÇÃO DO PERFIL DE VENTO MEDIDO POR RADIOSSONDAS E POR UM SODAR DURANTE O EXPERIMENTO DRYTOWET-AMC/LBA
}

\author{
CLÁUDIO MOISÉS SANTOS E SILVA ${ }^{1}$ ROBERTO FERNANDO DA FONSECA LYRA ${ }^{2}$ \\ ${ }^{1}$ Instituto Nacional de Pesquisas Espaciais / Centro de Previsão de Tempo e Estudos \\ Climáticos (INPE/CPTEC) \\ ${ }^{2}$ Universidade Federal de Alagoas, Centro de Ciência Exatas e Naturais, \\ Departamento de Meteorologia
}

claudio.moises@cptec.inpe.br, rffl@ccen.ufal.br

Recebido Setembro 2007 - Aceito Fevereiro 2009

\begin{abstract}
RESUMO
Foram comparados perfis do vento horizontal medidos por radiossondagens e por um equipamento SODAR durante experimento DRYTOWET-AMC/LBA. em 2002, no Estado de Rondônia. As análises foram feitas para três camadas, a saber: da superfície até $500 \mathrm{~m}$, de $500 \mathrm{~m}$ até $1000 \mathrm{~m}$ e de $1000 \mathrm{~m}$ até $1500 \mathrm{~m}$. Na primeira camada os campos apresentaram boa concordância, porém, a correlação entre as medidas diminuiu de forma significativa nas outras duas camadas. Os resultados sugerem que os dados do SODAR poderiam ser mais bem aproveitados se na instalação do instrumento fosse dada prioridade a uma camada menos profunda (até $500 \mathrm{~m}$ ) e com uma resolução vertical maior.
\end{abstract}

Palavras chaves: Amazônia, Camada Limite Atmosférica (CLA), medidas na CLA

\begin{abstract}
COMPARISON BETWEEN WIND PROFILES COLLECTED BY SODAR AND RADIOSONDES DURING THE DRYTOWET-AMC/LBA EXPERIMENT

The horizontal wind profiles measured by a SODAR and radiosondes during the DRYTOWET-AMC/ LBA experiment are compared. The analysis were made considering three layers: from surface up to $500 \mathrm{~m}$, from 500 up to $1000 \mathrm{~m}$ and from 1000 to $1500 \mathrm{~m}$. In the first layer the wind field profiles are in good agreement, however, the correlation values between SODAR and radiosonde data, gets significantly lower for the other two layers. The results suggest that the SODAR data should better be used if operating from surface up to $500 \mathrm{~m}$ and with greater vertical resolution.
\end{abstract}

Keywords: Amazônia, Atmospheric Boundary Layer, measurements into CLA

\section{INTRODUÇÃ̃O}

A Camada Limite Atmosférica (CLA) é a parte inferior da troposfera que sofre influência direta e responde às forçantes da superfície (arrasto friccional, evapotranspiração, transferência de calor sensível, emissão de poluentes e modificação do escoamento induzido pela terra), em uma escala de tempo de uma hora ou menos (Stull, 1988). Seibert et al. (2000) sumarizaram vários sistemas de coletas de dados no interior da CLA, dentre os quais as radiossondagens aparecem como a fonte mais comum de dados da estrutura vertical da baixa troposfera. As principais desvantagens da utilização de radiossondagens são o alto custo financeiro e baixa resolução vertical, apesar de atualmente já ser possível realizar radiossondagens com resolução espacial menor que $50 \mathrm{~m}$.
Outro instrumento de medidas comumente utilizado em estudos da CLA é o "Sound Detection and Ranging" (SODAR), o qual é uma sonda remota que processa o eco de um pulso acústico direcionado da superfície para a atmosfera. A mudança na freqüência do eco varia de acordo com a velocidade do vento (Efeito Doppler), enquanto a intensidade do eco varia de acordo com a intensidade e a estrutura térmica da turbulência atmosférica (Andrade et al., 2004). O pulso acústico emitido pela antena emissora do SODAR é re-emitido para a antena receptora através de espalhamento (caso seja um aparelho bi-estático, senão ele apresenta apenas uma antena, emissora/receptora). Esses elementos espalhados são não homogeneizados no campo de temperatura transportados pelo escoamento médio. As não-homogeneidades são associadas às térmicas (formadas pelo aquecimento da superfície) ou formadas por gradientes de 
temperatura, como ocorre em casos de inversão térmica. Dessa forma, o SODAR é capaz de identificar a altura da CLA durante o dia e a altura da Camada Residual (CR) no período noturno.

Diversos estudos verificaram a habilidade do SODAR em determinar a altura da inversão térmica (Beyrich, 1997; Crescenti, 1997; Keder, 1999; Seibert et al., 2000; Baumann \& Piringer, 2001; Asimakopoulos et al., 2004). Além desses, Andrade et al. (2004) realizaram estudos com um SODAR no Pantanal brasileiro, enquanto Silva (2005) verificou a confiabilidade desse instrumento em descrever a evolução da altura da Camada Limite Convectiva (CLC) em uma região desmatada na Amazônia. Em geral, esses estudos mostraram que os resultados do SODAR são bem correlacionados com aqueles obtidos por radiossondagens quando na ocorrência de fortes inversões térmicas. Contudo, dependendo do horário da comparação as correlações podem ser muito baixas. O SODAR também tem sido utilizado na caracterização de circulação de brisa terrestre e marítima (Childs \& Raman, 2005), poluição atmosférica (Schäfer et al., 2002), identificação de jatos de baixos níveis (Patra et al., 1998) e na dinâmica e determinação de parâmetros da CLA (Engelbart \& Bange, 2002; Tampieri \& Maurizi, 2003; Nair et al., 2004).

Durante o experimento de campo denominado DRY TO WET-2002 do projeto "Large Scale Biosphere-Atmosphere Experiment in Amazonia" (LBA), um SODAR operou concomitantemente com lançamentos de radiossondas. Segundo Seibert et al. (2000) uma grande vantagem do uso do SODAR é que ele opera de forma contínua e uma das informações deste aparelho é o perfil de vento. Poderiam, portanto, descrever a evolução de Jatos de Baixos Níveis (JBN) à Leste dos Andes, que atuam na região do Estado de Rondônia (Marengo et al., 2004); Jatos Noturnos (JN); e a estrutura dinâmica da CLA. Assim, para identificar possíveis aplicações das informações de vento extraído do SODAR, o presente trabalho tem por objetivo comparar o perfil vertical do vento obtido pelo SODAR com aquele obtido a partir de lançamentos de radiossondas durante o DRYTOWET-AMC/LBA.

\section{METODOLOGIA}

\section{1. Área de estudo}

A área de estudo (Fazenda Nossa Senhora - 1045'S, $62^{\circ} 22^{\prime} \mathrm{W}, 293 \mathrm{~m}$ ), fica localizada no município de Ouro Preto do Oeste, $50 \mathrm{~km}$ à Noroeste de Ji-Paraná no Estado de Rondônia. A Fazenda Nossa Senhora está centrada em uma região desmatada para práticas agropecuárias com aproximadamente $50 \mathrm{~km}$ de raio. A climatologia da região foi feita por Ferreira da Costa et al. (1998), utilizando-se de dez anos de dados de uma estação meteorológica convencional da Comissão Executiva do Plano de Lavoura Cacaueira (CEPLAC). Quanto ao ciclo diurno da precipitação, Tota et al. (2000) estudaram a precipitação ocorrida durante a estação chuvosa de 1999. Eles analisaram a precipitação segundo uma distribuição de freqüência e verificaram que apenas $1 \%$ da chuva era inferior a $1 \mathrm{~mm} \mathrm{~h}^{-1}$. Além desse limiar, os autores analisaram a distribuição das chuvas para eventos maiores que $1,3,5,10,15$ e $20 \mathrm{~mm} \mathrm{~h}^{-1}$. Notaram que cerca de $60 \%$ da precipitação era superior a $20 \mathrm{~mm}$ $\mathrm{h}^{-1}$, e que as maiores freqüências relativas ocorreram durante os intervalos de 2 a 4 HL (hora local) e 15 a $17 \mathrm{HL}$, sendo que esses eventos eram relacionados à sistemas convectivos de mesoescala e convecção local, respectivamente.

\subsection{SODAR}

O SODAR Doppler (Phased Array, Remtec, modelo PA2) utilizado na campanha pertence à Universidade de São Paulo (USP). Ele é monoestático (única antena receptora/emissora, cuja área é de $1,96 \mathrm{~m}^{2}$ ), funcionou com $10 \mathrm{~W}$ de potência e freqüência aproximada de $2 \mathrm{kHz}$. Os dados foram gravados em intervalos de 30 min e a altura máxima dos campos foi de 1,5 $\mathrm{km}$, com resolução vertical de $50 \mathrm{~m}$. A operação do SODAR foi do dia 12/09/2002 ao dia 03/11/2002. As variáveis analisadas neste estudo foram a componentes zonal (u) e meridional (v) e a magnitude do vento horizontal $(|\mathrm{V}|)$.

\subsection{Radiossondas}

As radiossondas utilizadas foram da marca Väisälä (RS80-15G, Helsinki, Finlândia). Esses instrumentos apresentam sensores de pressão, temperatura e umidade relativa e o campo de vento é inferido por telemetria através de um GPS. A taxa de amostragem foi de 10 segundos e a altura média de alcance ficou em torno de $20 \mathrm{~km}$. As radiossondagens foram realizadas entre 13/09/2002 a 03/11/2002, destacando-se dois períodos de coleta intensiva de dados. O primeiro foi do dia 24/09 a 29/09 (ainda no período seco) e o segundo de 15/10 a 29/10 (período chuvoso). $\mathrm{O}$ número de sondagens diárias não foi constante em todo o experimento, mas sempre que possível eram realizados às 02:00 HL, 08:00 HL, 11:00 HL, 14:00 HL, 17:00 HL e 20:00 HL. Nos outros dias a freqüência de lançamentos foi muito irregular, mas as sondagens das 08:00 HL, 14:00 HL e 20:00 HL eram realizadas prioritariamente. No total foram efetuadas 210 radiossondagens, as quais passaram por um controle de qualidade (Longo et al., 2002).

\subsection{Comparações}

A primeira dificuldade na comparação entre os dados foi quanto a diferença na resolução vertical. Enquanto no SODAR o 
espaçamento vertical era constante $(50 \mathrm{~m})$, nas radiossondagens esta distância depende da velocidade de ascensão do balão, a qual é função do conteúdo de gás utilizado. Tipicamente a velocidade de ascensão é ente 4 e $6 \mathrm{~m} \cdot \mathrm{s}^{-1}$ e como as radiossondas emitem informações a cada 10 segundos, então a resolução vertical fica entre 40 e 60 m. Por essas diferenças, foi feita uma interpolação linear nos dados das radiossondas para níveis regulares de $50 \mathrm{~m}$. O segundo passo foi identificar os perfis coincidentes para que fosse possível a realização de cálculos estatísticos com o mesmo número de amostras. Com este critério, embora houvesse 210 perfis de radiossondagens, somente 175 foram considerados, sendo 59 perfis a noite e 119 perfis de dia.

Determinado os pares para comparação foram efetuadas médias nas variáveis, dividindo-se o domínio vertical em três camadas de $500 \mathrm{~m}$. A Camada 1 (C1) de 0 a $500 \mathrm{~m}$, a Camada 2 (C2) de 500 a $1000 \mathrm{~m}$ e a terceira (C3) de 1000 a 1500 m. A partir daí calculou-se a média aritmética de cada série de dados, o coeficiente de correlação (r) e o viés. Este último determinado através da expressão:

$$
\text { vies }=\frac{1}{N} \sum_{i=1}^{N}\left(X_{i}-Y_{i}\right)
$$

sendo $\mathrm{N}$ o número de amostragens; $\mathrm{X}$ e $\mathrm{Y}$ as medidas do SODAR e radiossondas, respectivamente.

\section{RESULTADOS}

\subsection{Médias, desvios, viés e correlações}

A seguir serão comparadas as componentes zonal (u) e meridional (v), e o módulo da velocidade do vento ( $|\mathrm{V}|)$ obtidos a partir do SODAR e das radiossondas. Os índices 1, 2 e 3 são referentes as três camadas descritas no item 2.4. Os valores médios dessas variáveis, divididos por camadas e por período (dia e noite) são apresentados na Figura 1. Os dois sistemas de medidas apresentaram o escoamento meridional negativo (de Norte), o qual é resultado da circulação de grande escala. Nas três camadas, as medidas das radiossondas são maiores que as realizadas com o SODAR, sendo que a principal diferença foi na terceira camada e no período noturno $\left(1,4 \mathrm{~m} \cdot \mathrm{s}^{-1}\right)$. Já para o vento zonal os sinais dos dados só concordaram na primeira camada. Enquanto as radiossondas apresentaram valores negativos para as camadas 2 e 3, o SODAR indicou valores positivos e essa discrepância foi notada tanto durante o dia quanto à noite. No entanto, ressalta-se que a componente meridional possui maior intensidade do que a componente zonal.

A magnitude do vento do SODAR na primeira camada foi ligeiramente menor ao das radiossondas $\left(3,4 \mathrm{~m} . \mathrm{s}^{-1}\right.$ contra $\left.3,5 \mathrm{~m} . \mathrm{s}^{-1}\right)$; no entanto, nas camadas 2 e 3 a medida do SODAR foi relativamente maior, sendo a maior diferença na camada $2\left(2,2 \mathrm{~m} . \mathrm{s}^{-1}\right)$, o que representa cerca de $50 \%$ da velocidade registrada pelas radiossondas. Esse resultado concorda com o encontrado por Baumann \& Piringer (2001), no qual a medida do SODAR, para níveis mais elevados, tendeu a ser maior que as medidas feitas por balões cativos; entretanto, ressalta-se que o vento medido com auxílio de balões cativos e radiossondagens apresentem diferenças.

Os vieses e os coeficientes de correlação entre as medida são apresentados na Figura 2. Comparando as componentes, foi

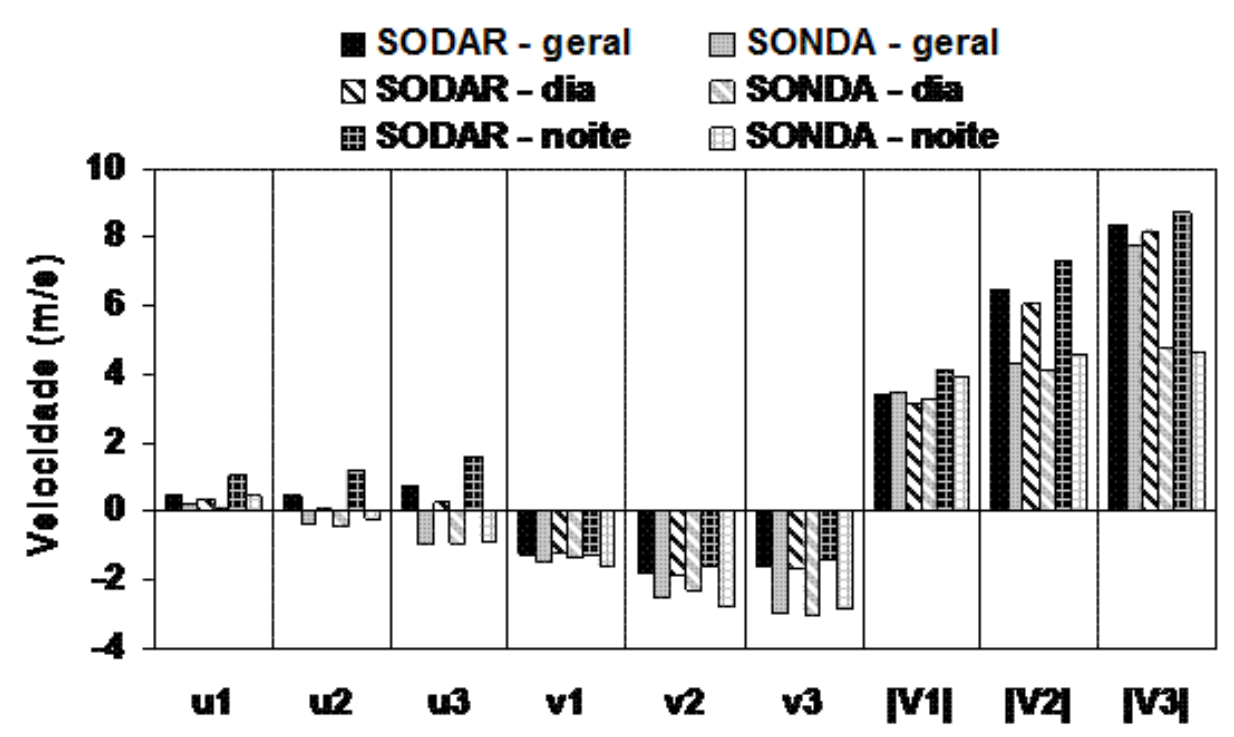

Figura 1 - Componentes zonal e meridional e magnitude da velocidade do obtidas pelo SODAR e por radiossondas durante o DRYTOWET-AMC/ LBA. Os índices 1, 2, são referentes as camadas descritas na item 2.4. 
observado que a componente zonal apresentou maiores vieses em todos os casos (dia e noite e por camadas). Por exemplo, no período noturno e terceira camada o viés foi de $2,5 \mathrm{~m} \cdot \mathrm{s}^{-1}$. Já o viés da componente meridional foi menor, embora a tendência de aumento com a altura fosse mantida. O viés do módulo da velocidade na primeira camada foi de $0,1 \mathrm{~m} \cdot \mathrm{s}^{-1}$, tanto para a noite quanto para o dia. Na camada 2 o viés foi de $2,7 \mathrm{~m} \cdot \mathrm{s}^{-1}\left(1,9 \mathrm{~m} \cdot \mathrm{s}^{-1}\right)$ durante a noite (dia); na terceira, foi de $4,7 \mathrm{~m} \cdot \mathrm{s}^{-1}\left(2,5 \mathrm{~m} \cdot \mathrm{s}^{-1}\right)$ a noite (dia). $\mathrm{O}$ coeficiente de correlação $\mathrm{r}$ diminui com a altura para todos os casos, sendo que as melhores correlações foram observadas para a componente meridional na primeira camada $(\mathrm{r}=0,87)$ e as piores foram para o módulo da velocidade na terceira camada $(-0,04)$.

\subsection{Estudo de Caso}

Os resultados até aqui mostrados compararam as medidas feitas por meio das radiossondagens e pelo SODAR. Foi possível notar que as medidas concordam principalmente na camada entre a superfície e $500 \mathrm{~m}$. Portanto, será feita uma breve descrição de um caso de ocorrência de JBN, e uma analise de quanto relevante podem ser as informações extraídas do SODAR nesta situação.

O caso estudado ocorreu na noite do dia 24 para o dia 25 de outubro de 2002. Na ocasião um JBN típico, como os descritos por Marengo et al. (2004) atuou na região. O campo de vento no nível de $850 \mathrm{hPa}$ (aproximadamente $1500 \mathrm{~m}$ ) das

GERAL $\triangle$ DIA \#NOITE
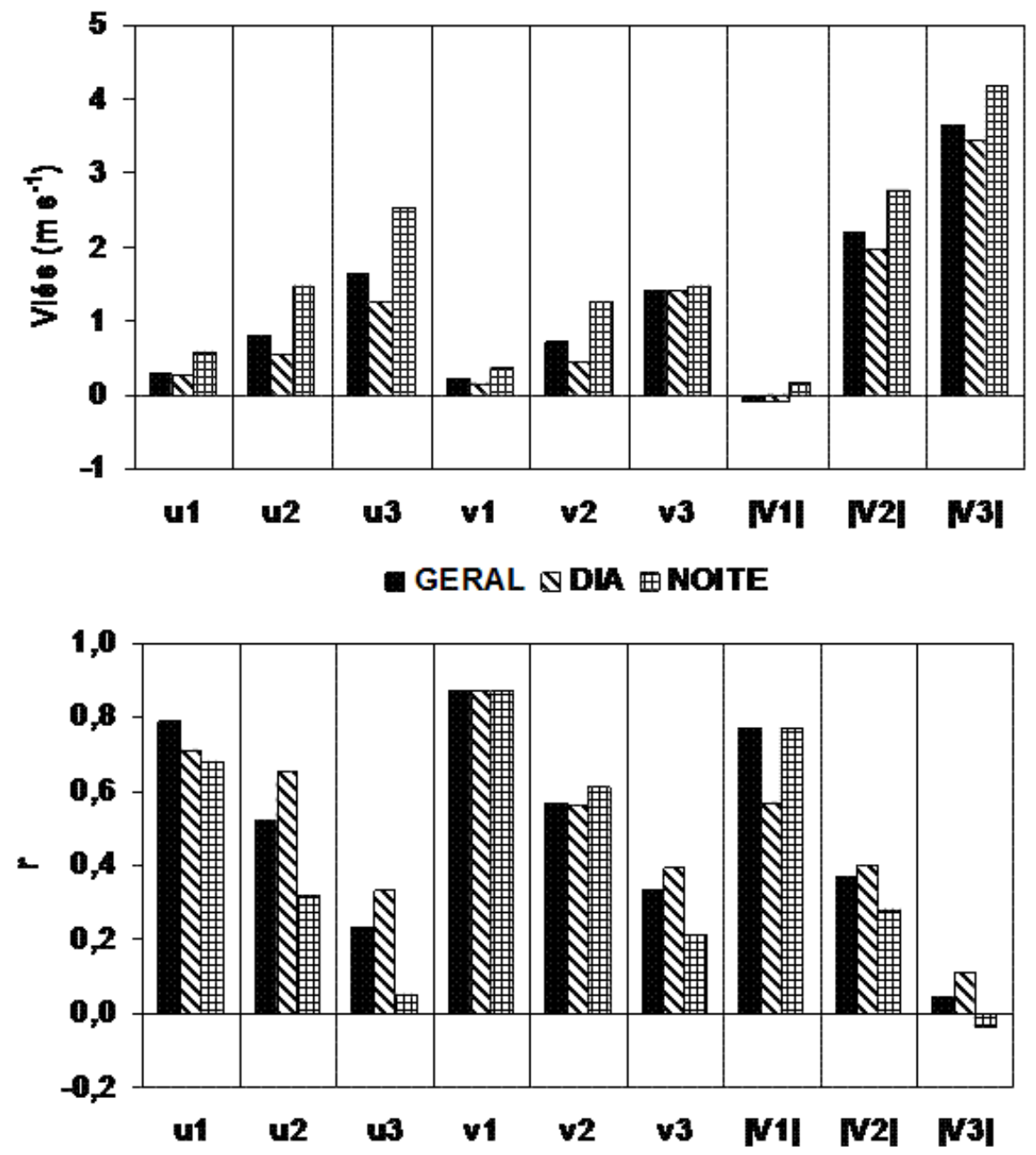

Figura 2 - Viés e coeficiente de correlação entre as medidas do SODAR e das radiossondas. 
reanálises do National Centers for Environmental Prediction (NCEP) é mostrado na Figura 3. É difícil comparar os dados de reanálises com medidas in situ como as de radiossondagens, pois a grade do modelo é de $2,5^{\circ} \times 2,5^{\circ}$ enquanto a medida é "pontual". Porém, na Figura 3 sobre a região do Estado de Rondônia (destacada com um círculo) a direção do vento era de Norte de 0600 às 1200 UTC (note que na região de estudo, $\mathrm{UTC}=\mathrm{HL}+4$ horas) e muda para Noroeste às 1800 UTC. Observando a Figura 4 (perfis de vento obtidos pelas radiossondagens e pelo SODAR), notou-se que a direção do vento em baixos níveis do modelo, parece concordar com as medidas das radiossondas.

Na Figura 4 são mostrados os perfis do vento obtidos a partir do lançamento de radiossondas e pelo SODAR. A primeira atenção é para as falhas de dados que eventualmente ocorreram nos lançamentos das radiossondas. Os perfis verticais das 0600 , 1200, 1500 e 1800 UTC são coincidentes, e mostram que os perfis não concordam muito bem para todos os níveis. Às 0600 UTC, até a altura de $500 \mathrm{~m}(\mathrm{C} 1)$ a direção do vento de Noroeste foi bem representada pelo SODAR, apesar da velocidade do vento ter sido superestimada em aproximadamente $30 \%$, ficando a velocidade média da camada medida pela radiossonda (SODAR) em torno de 5,0 m.s. ${ }^{-1}\left(6,8 \mathrm{~m} \cdot \mathrm{s}^{-1}\right)$. O mesmo ocorre nesta camada para os horários das 1200 e 1800 UTC, quando a direção do vento era de Norte e Oeste, respectivamente; porém, às 1500 UTC não houve concordância na direção do vento nesta camada. Na segunda camada as medidas são menos concordantes do que na primeira, e isso é mais crítico à medida que os níveis são elevados.

\section{DISCUSSÃO}

Os resultados apresentados indicam que o grau de confiabilidade das informações de vento obtido pelo SODAR diminui para camadas acima de $500 \mathrm{~m}$. Isso sugere que este equipamento poderia provir informações mais eficientes se fosse usado até esta altura, porém com maior resolução vertical. Essa afirmação concorda com o texto de Stull (1988), o qual argumentou que medidas com SODAR são pouco confiáveis para alturas acima de $1 \mathrm{~km}$, pois o pulso re-emitido para a antena receptora está sujeito a fortes interferências.

A relação entre a distância (2d) percorrida por um pulso
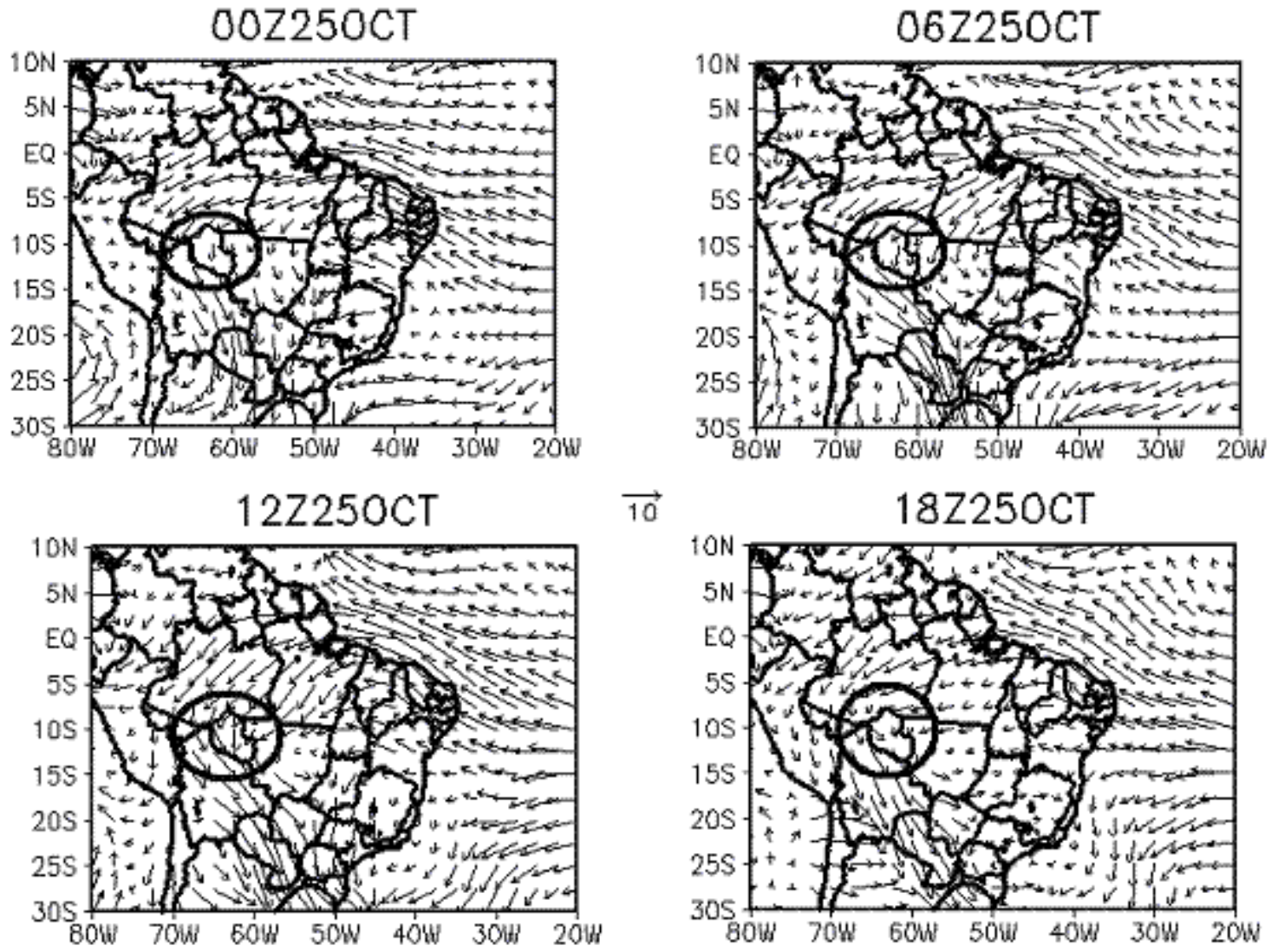

Figura 3 - Campo de vento obtidos das reanálises do NCEP no nível de 850 hPa para os horários de 0000, 0600, 1200 e 1800 UTC do dia 25 de outubro de 2002. A escala de velocidade é determinada pelo número $10 \mathrm{com}$ um vetor sobreposto no centro da figura. 
emitido pela antena do SODAR, a velocidade do som (c) e o tempo de retorno $(t)$ do pulso emitido segue as leis da mecânica newtoniana, $2 \mathrm{~d}=$ ct. Como c é constante e os pulsos re-emitidos são espalhados para qualquer $t>0$ (sendo $t=0 \mathrm{o}$ instante de emissão do pulso), então seria possível obter informações em maior resolução espacial se o intervalo entre as amostragens fosse diminuído.

Se fossem considerados somente os dados de vento até a altura de $500 \mathrm{~m}$, não seria possível (com o SODAR) estudar a estrutura dinâmica em toda a profundidade da CLC, principalmente após as $1100 \mathrm{HL}$, pois estudos prévios mostraram que a CLC sobre a região de estudo pode alcançar mais de $2 \mathrm{~km}$ de altura, principalmente na estação seca e em região de pastagem (Lyra et al., 2003; Fisch e al., 2004).

Entretanto, os resultados apresentados mostram que a confiabilidade do campo de vento do SODAR para além dessa altura é baixa (tomando as radiossondagens como referência), ou seja, parece mais interessante aproveitar o potencial do instrumento para a camada até a altura de $500 \mathrm{~m}$, mas direcionando os estudos com o SODAR para outras aplicações. Por exemplo, na análise da estrutura dinâmica da Camada Limite Noturna (CLN), inclusive para validar modelos de representação da estrutura e evolução da CLN como no estudo de Santos et al. (2007). Ademais, a transição na CLA de um regime de estabilidade (CLN) para um de instabilidade (CLC), comumente chamada de erosão da CLN (ver, por exemplo, Santos et al., 2003) poderia ser estudada dinamicamente em detalhes, algo que é difícil pela limitação de radiossondagens realizadas no início da manhã e pela falta de dados de balão cativo.

\section{CONCLUSÕES}

Foram usados dados de vento obtidos a partir de radiossondagens e por um SODAR para fins de comparação durante o experimento DRYTOWET-AMC/LBA, o qual ocorreu nos meses de Setembro e Outubro de 2002 no Estado de Rondônia. Foram analisadas as componentes zonal e meridional e a magnitude do vento em diferentes camadas (superfície a 500
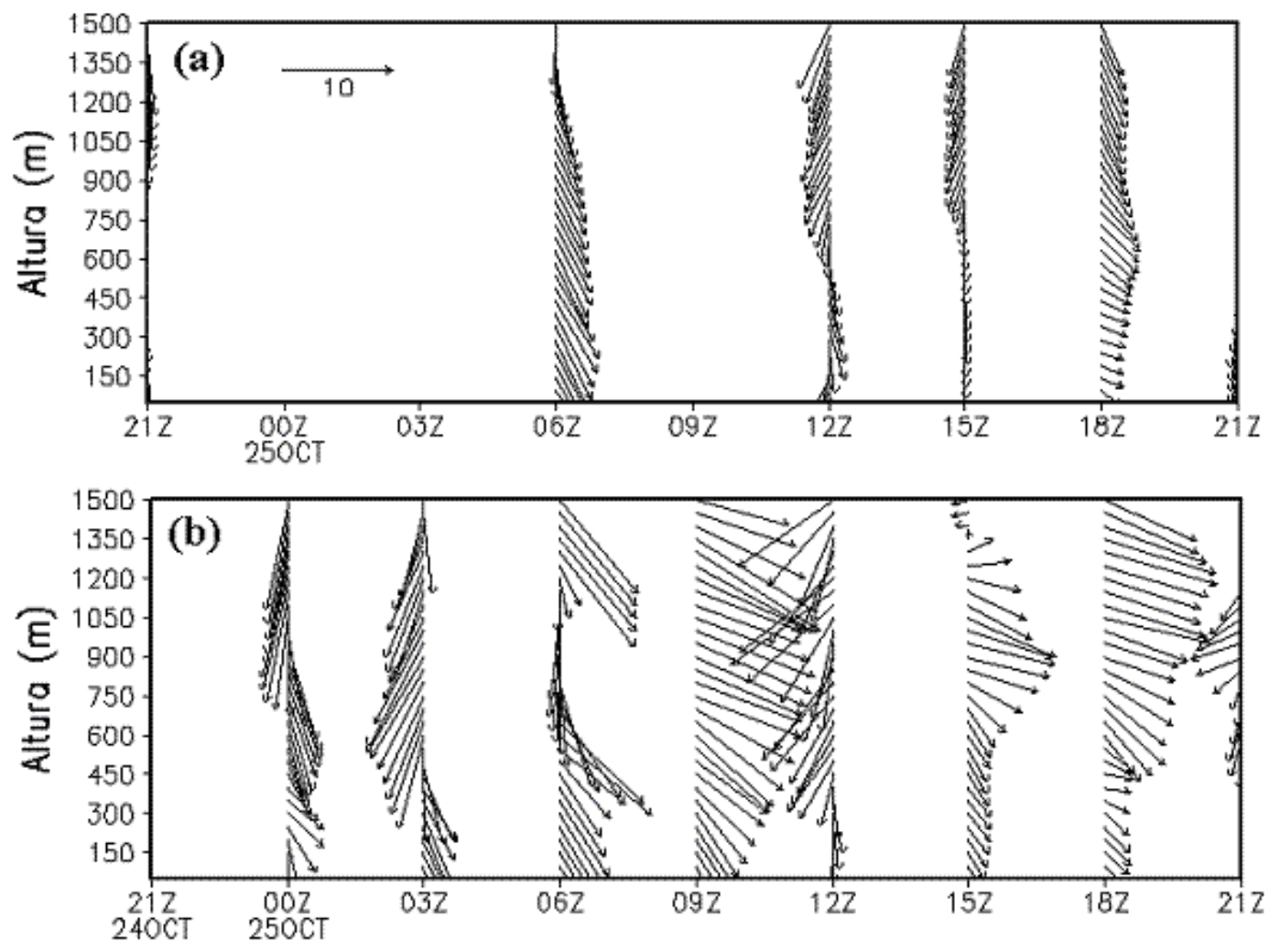

Figura 4 - Perfis verticais do vento horizontal obtidos pelas radiossondas (a) e pelo SODAR (b) para os dias 24 e 25 de outubro de 2002 . A escala de velocidade é determinada da mesma forma que na Figura 3. 
m, 500 a 1000 m e 1000 a 1500 m). Tomando como referência a medida das radiossondas, os resultados mostraram que o SODAR é um equipamento hábil em representar o perfil de vento até a altura de $500 \mathrm{~m}$; portanto, sendo importante na caracterização de Jatos Noturnos e erosão da CLN, por exemplo. Desta forma, sugere-se que as informações do SODAR sejam utilizadas com maior resolução espacial e menor domínio vertical.

\section{AGRADECIMENTOS}

A CAPES pela concessão de bolsa durante o curso de mestrado em meteorologia do primeiro autor na Universidade Federal de Alagoas. Os autores também agradecem a toda equipe que coletou os dados durante o experimento DRYTOWETAMC/LBA.

\section{REFERÊNCIAS BIBLIOGRÁFICAS}

ANDRADE, E. S.; SÁ, L.D. DE A.; JORGE, M.P.P.M. Estimativa da camada Limite atmosférica acima do Pantanal: Estudo comparativo usando radiossondagens e SODAR. In: XIII Congresso Brasileiro de Meteorologia (CD-ROM), Fortaleza, 2004.

ASIMAKOPOULOS, D.N.; HELMIS, C.G.; MICHOPOULOS, J. Evaluation of SODAR methods for the determination of the atmospheric boundary layer mixing height. Meteorology and Atmospheric Physics, v. 85, p. 85-92, 2004.

BAUMANN, K. \& PIRINGER, M. Two-years of boundary layer measurements with a SODAR-statistics and application. Physics and Chemistry of the Earth, Part B: Hydrology, Oceans and Atmosphere, v.26, p.205-211, 2001.

BEYRICH, F. Mixing Height Estimation from SODAR Data - A Critical Discussion. Atmospheric Environment. v. 31, p. 3941-3953, 1997.

CHILDS, P.P. \& RAMAN, S. Observations and Numerical Simulations of Urban Heat Island and Sea Breeze Circulations over New York City. Pure and Applied Geophysics, v. 162, p. 1955-1980, 2005.

CRESCENTI, G.H. A Look Back on Two Decades of Doppler Sodar Comparison Studies. Bulletin of the American Meteorological Society, v. 78, p. 651-673, 1997.

ENGELBART, D.A.M. \& BANGE, J. Determination of boundary-layer parameters using wind profiler/RASS and sodar/RASS in the frame of the LITFASS project. Theoretical and Applied Climatology, v. 73, p. 53-65, 2002.

FERREIRA DA COSTA, R.; FEITOSA, J.R.P.; FISCH, G.; SOUZA, S.S.; NOBRE, C.A. Variabilidade diária da precipitação em regiões de floresta e pastagem na Amazônia. Acta Amazônica, v.28, p.395-408, 1998.

FISCH, G.; TOTA DA SILVA, J.; MACHADO, L.A.T.; SILVA
DIAS, M.A.F.; LYRA, R.F.F.; NOBRE, C.A.; DOLMAN, A.J.; GASH, J.H.C. The convective boundary layer over pasture and forest in Amazonia. Theoretical and Applied Climatology, v.78, p.47-59, 2004.

KEDER, J. Detection of inversions and mixing height by REMTECH PA2 SODAR in comparison with collocated radiossonde measurements. Meteorology and Atmospheric Physics, v. 71, p. 133-138, 1999.

LONGO, M.; ALBRECHT, R.I.; MACHADO, L.A.T.; FISCH, G.; SILVA DIAS, M.A.F. Controle de qualidade dos dados de radiossondagens da campanha WET-AMC/LBA. Revista Brasileira de Meteorologia, v.17, p. 243-253, 2002.

LYRA, R.F.F.; MOLION, L.C.B.; SILVA, M.R.G.DA.; FISCH, G.; NOBRE, C.A. Some aspects of the atmospheric boundary layer over western Amazonian: Dry season 1994. Revista Brasileira de Meteorologia, v.18, p. 79-85, 2003.

MARENGO, J.A.; SOARES, W.S.; SAULO, C.; NICOLINI, M. Climatology of the Low-Level Jet East of the Andes as Derived from the NCEP-NCAR Reanalyses: Characteristics and Temporal Variability. Journal of Climate, v. 17, p. 22612280, 2004.

NAIR, K.N.; FREITAS, E.D.; SANCHEZ-CCLOYLLO, O.R.; SILVA DIAS, M.A.F.; SILVA DIAS, P.L.; ANDRADE, M.F.; MASSAMBANI, O. Dynamics of urban boundary layer over São Paulo associated with mesoscale processes. Meteorology and Atmospheric Physics, v. 86, p. 87-92, 2004.

PATRA, A. K.; DE, U. K.; LOHAR, D. Existence of Low level Jet during Premonsoon Period over Eastern India and its Role in the Initiation of Nocturnal Thunderstorms. Atmosfera, v. 12, p.15-26, 1998.

SANTOS, R.M.N.; FISCH, G.; DOLMAN, A.J. Erosão da Camada Limite Noturna sobre a Amazônia: aspectos observacionais. Ciência e Natura, v. esp., p. 291-296, 2003.

SANTOS, R.M.N.; FISCH, G.; DOLMAN, A.J.; WATERLOO, M. Modelagem da Camada Limite Noturna (CLN) durante a época úmida na Amazônia, sob diferentes condições de desenvolvimento. Revista Brasileira de Meteorologia, v. 22, p. 387-407, 2007.

SEIBERT, P.; BEYRICH, F.; GRYNING, S.E.; JOFFRE, S.; RASMUSSEN,A. TERCIER, P. Review and intercomparision of operational methods for the determination of the mixing height. Atmospheric Environment, v.34, p.1001-1027, 2000.

SCHÄFER, K.; FÖMMEL, G.; HOFFMANN, H.; BRIZI, S.; JUNKERMANN, W.; EMEIS, S.; JAHN, C.; LEIPOLD, S.; SEDLMAIER, S.; DINEV2, S.; REISHOFER, G.; WINDHOLZ, L.; SOULAKELLIS, N.; SIFAKIS, N.; SARIGIANNIS, D. Three- dimensional ground-based measurements of urban air quality to evaluate satellite 
derived interpretations for urban air pollution. Water, Air, and Soil Pollution: Focus, v. 2, p. 91-102, 2002.

SILVA, C.M.S. Comportamento da Camada Limite Atmosférica sobre pastagem em Rondônia durante a transição da estação seca para chuvosa em 2002. Alagoas, 2005. Dissertação (mestrado em Meteorologia). UFAL. 82p.

STULL, R. B. An introduction to boundary Layer meteorology. Dordrecht: Kluwer, 1988. 666p.
TAMPIERE, F. e MAURIZI, A. Investigations on convective boundary layer turbulence using SODAR data. Annals of Geophysics, v. 46, p. 451-457, 2003.

TOTA, J.; FISCH, G.; FUENTES, J.; OLIVEIRA, P.J.; GARSTANG, M.; HEITZ, R.; SIGLER, J. Análise da variabilidade diária da precipitação em área de pastagem para a época chuvosa de 1999 - projeto TRMM/LBA. Acta Amazônica, v. 30, p. 629-639, 2000. 